\title{
HDAC4 promotes nasopharyngeal carcinoma progression and serves as a therapeutic target
}

\author{
Chun Cheng ${ }^{1}$, Jun Yang ${ }^{1}$, Si-Wei Li ${ }^{2}$ Guofu Huang ${ }^{1}$, Chenxi Li', Wei-Ping Min ${ }^{3}$ and Yi Sang (D)
}

\begin{abstract}
Histone deacetylases (HDACs) are involved in tumor progression, and some have been successfully targeted for cancer therapy. The expression of histone deacetylase 4 (HDAC4), a class Ila HDAC, was upregulated in our previous microarray screen. However, the role of HDAC4 dysregulation and mechanisms underlying tumor growth and metastasis in nasopharyngeal carcinoma (NPC) remain elusive. Here, we first confirmed that the HDAC4 levels in primary and metastatic NPC tissues were significantly increased compared with those in normal nasopharyngeal epithelial tissues and found that high HDAC4 expression predicted a poor overall survival (OS) and progression-free survival (PFS). Functionally, HDAC4 accelerated cell cycle G1/S transition and induced the epithelial-to-mesenchymal transition to promote NPC cell proliferation, migration, and invasion in vitro, as well as tumor growth and lung metastasis in vivo. Intriguingly, knockdown of $\mathrm{N}-\mathrm{CoR}$ abolished the effects of HDAC4 on the invasion and migration abilities of NPC cells. Mechanistically, HDAC3/4 binds to the E-cadherin promoter to repress E-cadherin transcription. We also showed that the HDAC4 inhibitor tasquinimod suppresses tumor growth in NPC. Thus, HDAC4 may be a potential diagnostic marker and therapeutic target in patients with NPC.
\end{abstract}

\section{Introduction}

Nasopharyngeal carcinoma (NPC), originating from the nasopharynx epithelium, is highly prevalent in East and Southeast Asia, particularly in Southern China ${ }^{1}$. NPC is a poorly differentiated cancer and has the highest metastasis rate among head and neck cancers ${ }^{2}$. Through the combined use of intensity-modulated radiotherapy (IMRT), concurrent chemoradiotherapy, and magnetic resonance imaging $(\mathrm{MRI})^{3}$, locoregional control has steadily improved. However, distant metastasis remains the major cause of treatment failure in patients with $\mathrm{NPC}^{4}$. Furthermore, the molecular mechanisms underlying NPC growth and metastasis remain largely

\footnotetext{
Correspondence: Yi Sang (ndsfy001889@ncu.edu.cn)

'Jiangxi Key Laboratory of Cancer Metastasis and Precision Treatment,

Department of Center Laboratory, The Third Affiliated Hospital of Nanchang University, Nanchang 330008, China

${ }^{2}$ Department of Oncology, Tongji Huangzhou Hospital of Huazhong University of Science and Technology, Hubei, People's Republic of China

Full list of author information is available at the end of the article

These authors contributed equally: Chun Cheng, Jun Yang, Si-Wei Li

Edited by M. Agostini
}

unknown. Therefore, effective therapeutic strategies are needed to treat NPC patients.

In our previous study, we used a microarray containing whole human transcription factors to screen a pair of NPC primary tumor tissues and metastatic tissues in lymph nodes (LNs) and two cell lines (S18 and S26) derived from the NPC cell line CNE2 with high and low metastatic abilities, respectively ${ }^{5}$. TEL2 was one of the most altered transcription factors among the downregulated genes ${ }^{2,5}$; however, the upregulated genes were not explored in our previous study. Importantly, histone deacetylase 4 (HDAC4) expression increased simultaneously in LN metastases compared with primary tissues and in S18 cells compared with S26 cells in these microarray results.

Based on sequence homologies, the 18 human HDACs are classified into four groups: class I, class II, class III, and class IV HDACs ${ }^{6}$. HDAC4 is a member of the class IIa HDACs and is similar to the other class IIa members (HDAC5, HDAC7, and HDAC9), which regulate gene expression by mediating different signaling pathways ${ }^{7,8}$.

\section{(-) The Author(s) 2021}

(c) (i) Open Access This article is licensed under a Creative Commons Attribution 4.0 International License, which permits use, sharing, adaptation, distribution and reproduction cc) in any medium or format, as long as you give appropriate credit to the original author(s) and the source, provide a link to the Creative Commons license, and indicate if changes were made. The images or other third party material in this article are included in the article's Creative Commons license, unless indicated otherwise in a credit line to the material. If material is not included in the article's Creative Commons license and your intended use is not permitted by statutory regulation or exceeds the permitted use, you will need to obtain permission directly from the copyright holder. To view a copy of this license, visit http://creativecommons.org/licenses/by/4.0/. 
Recently, accumulating evidence has shown that HDAC4 overexpression is correlated with the development and progression of cancers, including multiple myeloma, prostate cancer, breast cancer, colon cancer, glioblastoma, and esophageal cancer $^{9-14}$. Elevated HDAC4 expression plays an oncogenic role via different mechanisms that depend on the cellular context and activated downstream signaling pathways ${ }^{8}$. For example, HDAC4 inhibits HOXB13 transcription to affect cell growth in androgen receptor (AR)-negative prostate cancers ${ }^{15}$, but the HDAC4-RelB-p52 complex maintains a repressive chromatin state around the proapoptotic genes Bim and BMF and regulates multiple myeloma survival and growth ${ }^{16}$. However, the function of HDAC4 and underlying molecular mechanism in NPC are poorly understood.

In the present study, we elucidated the function and underlying molecular mechanisms of HDAC4 in NPC. Our data showed that HDAC4 promotes NPC cell proliferation, invasion, and migration in vitro and tumor growth and lung metastasis in vivo. In addition, HDAC4 promotes the cell cycle and induces the epithelial-tomesenchymal transition (EMT). Knockdown of N-CoR abolished the effects of HDAC4 on the invasion and migration abilities of NPC cells. Finally, the HDAC4 inhibitor tasquinimod was used to target NPC cells with high HDAC4 expression, suggesting a promising therapeutic strategy.

\section{Materials and methods}

\section{Cell lines and reagents}

Four NPC cell lines (S26, S18, 6-10B, and 5-8F) were cultured in Dulbecco's modified Eagle's medium (DMEM; Gibco; Thermo Fisher Scientific, Inc., Waltham, MA, USA) supplemented with 10\% fetal bovine serum (Gibco; Thermo Fisher Scientific, Inc.) and maintained at $37^{\circ} \mathrm{C}$ in an incubator containing $5 \% \mathrm{CO}_{2}$. The normal nasopharyngeal epithelial cell line (NP69) was grown in definedKSFM medium supplemented with epidermal growth factor (EGF) (Invitrogen, Carlsbad, USA). The five cell lines were obtained from Sun Yat-sen University Cancer Center (SYSUCC) ${ }^{2}$. S18 and S26 were derived from the NPC cell line CNE2 with high and low metastatic ability, respectively. 5-8F and 6-10B were derived from the NPC Sune1 cell line with high and low metastatic ability, respectively ${ }^{4}$. HEK-293T cells were obtained from the ATCC and cultured in DMEM (Gibco; Thermo Fisher Scientific, Inc., Waltham, MA, USA). All of the cells were authenticated using short-tandem repeat profiling, tested for mycoplasma contamination, and cultured for $<2$ months.

\section{Plasmids}

The full-length CDSs of human HDAC4 was cloned into the pSin-puro vector. shRNAs were established using the Sigma-Aldrich shRNA system (Merck KGaA) according to the manufacturer's protocols. Scr shRNA (with no known targets in the human genome) had the sequence 5'-GGGCGAGGAGCTGTTCACCG-3' , and the oligonucleotides for human HDAC4 shRNA\#1 and \#2 were $5^{\prime}$-GTTACAAGAATTTGTCCTCAA- $3^{\prime}$ and $5^{\prime}$ CGACTCATCTTGTAGCTTATT- $3^{\prime}$, respectively. All the recombinant plasmids were verified by DNA sequencing (Ruiboxingke Biotech Co., Ltd., Beijing, China).

\section{Antibodies}

Human anti-HDAC4 (cat. no. 7628) and anti-HDAC3 (cat. no. 85057) were obtained from Cell Signaling Technology. Human anti-N-CoR (cat. no. ab24552) was obtained from Abcam. Anti- $\beta$-actin (cat. no. 60008-1) was obtained from ProteinTech Group, Inc. (Chicago, IL, USA). The epithelial-mesenchymal transition (EMT) Antibody Sampler Kit (anti-E-cadherin, anti-N-cadherin, anti-Slug, and anti-Snail) (cat. no. 9782), anti-Cyclin D1 (cat. no. 2978), anti-CDK4 (cat. no. 12790), and antiCDK6 (cat. no. 13331) were obtained from Cell Signaling Technology. Human anti-HDAC4 (cat. no. sc-46672) was obtained from Santa Cruz Biotechnology for ChIP analysis.

\section{Stable lines}

For the knockdown of HDAC4 in 5-8F and S18 cells, $3 \mu \mathrm{g}$ of shRNA-Scr or $3 \mu \mathrm{g}$ of shRNA-HDAC4\#1 or \#2 was co-transfected with $3 \mu \mathrm{g}$ of pMD2.G and $3 \mu \mathrm{g}$ of psPAX2 into $293 \mathrm{~T}$ cells for $48 \mathrm{~h}$ using Lipofectamine 2000. The recombinant viruses were subsequently collected and added to S18 and 5-8F cells, which were then cultured with $8 \mu \mathrm{g} / \mathrm{ml}$ of polybrene for $24 \mathrm{~h} .5-8 \mathrm{~F}$ and S18 cells were infected with recombinant viruses at a multiplicity of infection (MOI) of 10. For the overexpression of HDAC4 in S26 and 6-10B cells, $3 \mu \mathrm{g}$ of pSin-puro delivering HDAC4 or $3 \mu \mathrm{g}$ of the empty vector was co-transfected with $3 \mu \mathrm{g}$ of pMD2.G and $3 \mu \mathrm{g}$ of psPAX2 into HEK-293T cells for $48 \mathrm{~h}$ using Lipofectamine 2000 . The recombinant viruses were subsequently collected and added to S26 and 6-10B cells, which were then cultured with $8 \mu \mathrm{g} / \mathrm{ml}$ of polybrene for $24 \mathrm{~h}$. S26 and 6-10B cells were infected with recombinant viruses at an $\mathrm{MOI}$ of 5 . The stable lines were selected with $1 \mu \mathrm{g} / \mathrm{ml}$ of puromycin for 2 weeks.

\section{RNA extraction and qRT-PCR}

These procedures were performed as previously described $^{5,17}$. Briefly, total RNA was isolated using TRI$\mathrm{zol}^{\circledR}$ reagent (Invitrogen; Thermo Fisher Scientific, Inc.) according to the manufacturer's instructions. First-strand cDNA was synthesized via reverse transcription using the Revert Aid $^{\mathrm{TM}}$ First Strand cDNA Synthesis Kit (cat. no. 6210A; TaKaRa Bio, Inc., Otsu, Japan). Subsequently, 
qRT-PCR was performed using a CFX96 Real-Time PCR Detection System (Bio-Rad Laboratories, Inc., Hercules, CA, USA) and SYBR ${ }^{\circledR}$ Green mix (Tiangen Biotech Co., Ltd., Beijing, China). The qRT-PCR thermal cycling conditions were initiated using a denaturation step at $95^{\circ} \mathrm{C}$ for $15 \mathrm{~min}$ and comprised 40 cycles (denaturation at $95^{\circ} \mathrm{C}$ for $15 \mathrm{~s}$, annealing at $60^{\circ} \mathrm{C}$ for $30 \mathrm{~s}$ and elongation at $72^{\circ} \mathrm{C}$ for $30 \mathrm{~s}$ ). The amplification products were analyzed using the $2^{-\Delta \Delta C q}$ method $^{18}$, and the expression levels in each sample were calibrated to those of the housekeeping gene GAPDH. The primers employed for amplifying HDAC4, E-cadherin, N-cadherin, Snail, Slug, and GAPDH are listed in Supplementary Table 1.

\section{RNA interference}

An effective siRNA oligonucleotide targeting N-CoR, $5^{\prime}$ AAUGCUACUUCUCGAGGAAACA-3', was synthesized by Guangzhou RiboBio Co., Ltd. (Guangzhou, China). Approximately $2.5 \times 10^{5} \mathrm{NPC}$ cells/well were seeded in 6well culture plates the day before transfection. Transfection was performed according to the manufacturer's instructions, and the cells were transfected with $50 \mathrm{nM}$ of siRNA using Lipofectamine RNAiMAX transfection reagent (Invitrogen; Thermo Fisher Scientific, Inc.).

\section{Cell proliferation assay}

In vitro cell proliferation was assessed using the Cell Counting Kit-8 (CCK8) assay. For cell proliferation, the cells were seeded in 96-well plates at a density of 1000 cells/well and incubated for $1,2,3,4$, or 5 days; $10 \mu$ of CCK 8 reagent (Beyotime Institute of Biotechnology, Haimen, China) was then added to each well, and the plate was incubated for $1.5 \mathrm{~h}$ at $37^{\circ} \mathrm{C}$. The absorbance value (optical density) of each well was measured at $450 \mathrm{~nm}$ using an iMark microplate reader (Bio-Rad Laboratories, Inc.).

\section{Colony formation assay}

The cells in this study were plated in six-well culture plates at a density of $5 \times 10^{2}$ cells/well. Each group included three wells. The cells were incubated for 15 days at $37^{\circ} \mathrm{C}$, washed twice with PBS, incubated with methyl alcohol for $15 \mathrm{~min}$, and stained with $0.1 \%$ crystal violet for $60 \mathrm{~min}$. Clusters were assessed by light microscopy, and each cluster containing $\geq 50$ cells was counted as a colony.

\section{Transwell assays}

For the Transwell migration assay, $200 \mu \mathrm{l}$ of serum-free DMEM containing either $4.0 \times 10^{4}$ (S18 and 5-8 F) or $8 \times$ $10^{4}$ (S26 and 6-10B) cells was added to cell culture inserts with an $8-\mu \mathrm{m}$ microporous filter without an extracellular matrix coating (BD Biosciences, Franklin Lakes, NJ, USA). DMEM containing 10\% fetal bovine serum (FBS; Gibco;
Thermo Fisher Scientific, Inc.; cat. no. 10270-106) was then added to the bottom chamber. After $24 \mathrm{~h}$ of incubation at $37^{\circ} \mathrm{C}$ in $5 \% \mathrm{CO}_{2}$, the cells on the lower surface of the filter were fixed, stained and examined under a light microscope. The number of migrated cells in three random optical fields (magnification, $\times 10$ ) from triplicate filters was averaged. For the Transwell invasion assay, $8.0 \times 10^{4}$ (S18 and 5-8F) or $1.6 \times 10^{5}$ (S26 and 6-10B) cells resuspended in $200 \mu \mathrm{l}$ serum-free DMEM were added to cell culture inserts, which contained $8-\mu \mathrm{m}$ microporous filters and were coated with Matrigel (BD Biosciences; cat. no. 354480). DMEM containing 10\% FBS was then added to the bottom chamber. After $24 \mathrm{~h}$ of incubation at $37^{\circ} \mathrm{C}$ in $5 \% \mathrm{CO}_{2}$, the cells on the lower surface of the filter were fixed with methyl alcohol for $15 \mathrm{~min}$ at room temperature, stained with $0.1 \%$ crystal violet for $60 \mathrm{~min}$ at room temperature, and examined under a light microscope. The number of invading cells in three random optical fields (magnification, $\times 100$ ) from triplicate filters was averaged.

\section{Cell cycle analysis}

These procedures were performed as previously described ${ }^{19}$. Briefly, cells were harvested and fixed with $70 \%$ ethanol at $4{ }^{\circ} \mathrm{C}$ for $1 \mathrm{~h}$ overnight. Next, the cells were stained with PI/RNase and staining buffer at $4{ }^{\circ} \mathrm{C}$ for $30 \mathrm{~min}$, followed by analysis using a flow cytometer (BD Biosciences, USA).

\section{Western blotting and immunoprecipitation}

Western blotting procedures were performed as described previously ${ }^{20,21}$. Briefly, cells were collected and lysed in RIPA buffer (150 mM NaCl, 0.5\% EDTA, $50 \mathrm{mM}$ Tris, $0.5 \%$ NP40) at $4{ }^{\circ} \mathrm{C}$ for $30 \mathrm{~min}$ and centrifuged for $20 \mathrm{~min}$ at $12,000 \mathrm{rpm}$ at $4{ }^{\circ} \mathrm{C}$. The lysates were obtained, and the protein concentration was determined using the BCA method. Protein extracts were separated by $10 \%$ SDS-PAGE at a voltage of $120 \mathrm{~V}$ for $2 \mathrm{~h}$ at room temperature, transferred to PVDF membranes with an electrical current of $350 \mathrm{~mA}$ at $4{ }^{\circ} \mathrm{C}$ for $2 \mathrm{~h}$, and blocked with $5 \%$ non-fat milk for $1 \mathrm{~h}$ at room temperature. The membranes were probed with antibodies against HDAC4, E-cadherin, N-cadherin, Snail, Slug, and $\beta$-actin. The blots were incubated with peroxidaseconjugated secondary antibodies against rabbit (1:20,000; 401BW, Promega Corporation, Madison, WI, USA) and mouse (1:20,000; W402B, Corporation, Madison, WI, USA), detected using an ECL chemiluminescence system (cat. no. P0018F; Beyotime, China) and exposed to radiographic film (Carestream, Catalog Number 6535876). To analyze the endogenous interaction among HDAC4, N-CoR, and HDAC3 using immunoprecipitation, the clarified supernatants were first incubated with anti-HDAC4, anti-N-CoR, and anti-HDAC3 for $2 \mathrm{~h}$ at $4{ }^{\circ} \mathrm{C}$. Next, protein A/G-agarose 
was added from $2 \mathrm{~h}$ to overnight, and the precipitates were washed four times with RIPA buffer and analyzed by western blotting.

\section{Luciferase assay}

The assay was carried out as described previously ${ }^{17}$. Briefly, 6-10B cells were seeded in 12-well plates at a density of $3 \times 10^{5}$ cells/well and transfected with $0.8 \mu \mathrm{g}$ of a promoter-luciferase reporter plasmid. The cells were cotransfected with $8 \mathrm{ng}$ of Renilla luciferase plasmid as an internal control. After transfection for $48 \mathrm{~h}$, the luciferase activity was measured using the Dual-Luciferase Reporter Assay Kit (Promega, Madison, WI, USA). Three independent experiments were performed.

\section{ChIP assays}

These procedures were performed as previously described $^{2,5,20}$ using a ChIP kit (cat. no. 53008, Active Motif, Carlsbad, CA, USA). Briefly, once the cells reached $80 \%$ confluence, Complete Cell Fixative Solution (included in the kit) was added to the existing culture medium at room temperature to fix the cells, and then the fixation reaction was stopped by adding Stop Solution (included in the kit) to the culture medium. The cells were collected by centrifugation at $1000 \times g$ for $5 \mathrm{~min}$ at $4{ }^{\circ} \mathrm{C}$. Subsequently, the nuclear pellet was resuspended in ChIP Buffer (included in the kit). DNA in the cell lysate was sheared using a sonication instrument (Ningbo Scientz Biotechnology Co., Ltd., Ningbo, China) to 200- to 500-bp-long fragments. Total genomic DNA (input) was quantified, and $20 \mu \mathrm{g}$ of chromatin from each sample was immunoprecipitated overnight at $4{ }^{\circ} \mathrm{C}$ with $5 \mu \mathrm{g}$ of anti-HDAC3, anti-HDAC4, or normal IgG as a negative control. Next, nucleosome complexes were isolated with protein $\mathrm{G}$-agarose beads for $3 \mathrm{~h}$ at $4{ }^{\circ} \mathrm{C}$. Bound DNA-protein complexes were eluted, and cross-links were reversed after a series of washes using the washing reagent provided in the ChIP kit. Purified DNA was resuspended in TE buffer. Subsequently, PCR was performed using PrimeSTAR® Max DNA Polymerase (cat. no. R045A, TaKaRa Bio, Inc.). The qRT-PCR thermal cycling conditions comprised a denaturation step at $94^{\circ} \mathrm{C}$ for $2 \mathrm{~min}$, followed by 35 cycles of denaturation at $98^{\circ} \mathrm{C}$ for $10 \mathrm{~s}$, annealing at $60^{\circ} \mathrm{C}$ for $15 \mathrm{~s}$ and elongation at $72{ }^{\circ} \mathrm{C}$ for 30 s). The primers are listed in Supplementary Table 1.

\section{Molecular docking}

The crystal structures of HDAC1 (PDB: 6Z2J), HDAC2 (PDB: 5IX0), HDAC3 (PDB: 4A69), HDAC4 (PDB: 4CBT), HDAC6 (PDB: 6CED), HDAC7 (PDB: 3ZNR), and HDAC8 (PDB: 5FCW) were obtained from the RCSB Protein Data Bank. The structural formula of the HDAC4 inhibitor tasquinimod was obtained from PubChem Compound. Ligand docking studies were performed using Maestro, Schrödinger (v11.9).

\section{Animal experiments}

All the animal studies were performed in accordance with protocols approved by Nanchang University (Nanchang, China). The mice were maintained under specific pathogen-free conditions at a temperature of $20-25^{\circ} \mathrm{C}$ and $50-70 \%$ humidity, under a light/dark cycle of $12 \mathrm{~h}$, with free access to water and food. In total, 108 4-weekold male athymic nude mice were obtained from the Shanghai Institutes for Biological Sciences, Chinese Academy of Sciences (Shanghai, China). In each animal experiment, the mice were randomly assigned to each group. For subcutaneous injection, $2 \times 10^{6}$ cells were mixed with $0.2 \mathrm{ml}$ of PBS (pH 7.4) and 30\% (v/v) Matrigel matrix (BD Biosciences). The suspensions were injected subcutaneously into the flanks of 4-week-old male athymic nude mice, which were monitored over 5 weeks $(n=$ 6 per group). For tail vein injection, $3 \times 10^{6}$ cells were resuspended in $200 \mu \mathrm{l}$ of PBS (Biological Industries, Beit Haemek, Israel) and injected into the lateral tail vein of mice that did not receive a subcutaneous injection of cells $\left(3 \times 10^{6}\right.$ cells/animal). Mice were sacrificed 8 weeks following injection, and nodules were counted. To analyze the antitumor activity of tasquinimod on tumor growth, mice received tasquinimod $(10 \mathrm{mg} / \mathrm{kg})$ or vehicle $(0.1 \%$ DMSO) every 3 days via intragastric administration.

\section{IHC and histological evaluation}

IHC analysis was performed as described previously,21. Briefly, samples were fixed in $10 \%$ formalin for $10 \mathrm{~h}$ at room temperature and embedded in paraffin. Sections $(3-\mu \mathrm{m}$ thick) were prepared and mounted onto positively charged glass slides. Sections for HDAC4 staining were incubated in $10 \mathrm{mM}$ citrate buffer (pH 6.0) and boiled in a microwave oven for $15 \mathrm{~min}$. The sections were incubated with primary antibodies against HDAC4 at a 1:250 dilution at $4{ }^{\circ} \mathrm{C}$ overnight in a humidified container, and isotype-matched IgG was used as a negative control. IHC staining was evaluated by two independent pathologists specializing in NPC. The expression level of HDAC4 was scored using the semiquantitative immunoreactive score (IRS) scale described by Remmele and Stegner ${ }^{22}$. Briefly, the HDAC4 signal was detected in the cytoplasm and nucleus. The staining intensity (SI) for HDAC4 has four classes in NPC tissues: 0 , absent; 1 , weak; 2 , moderate; and 3, strong. The percentage of stained cells was categorized as follows: 0 , no staining; 1 , $1-10 \%$; $2,11-50 \%$; $3,51-80 \%$; and 4, 81-100\% stained cells. The intensity and proportion scores were then multiplied to obtain a final score.

\section{Study approval}

The use of human NPC tissues was reviewed and approved by the Ethical Committee of The Third Affiliated Hospital of Nanchang University (Nanchang, China). 


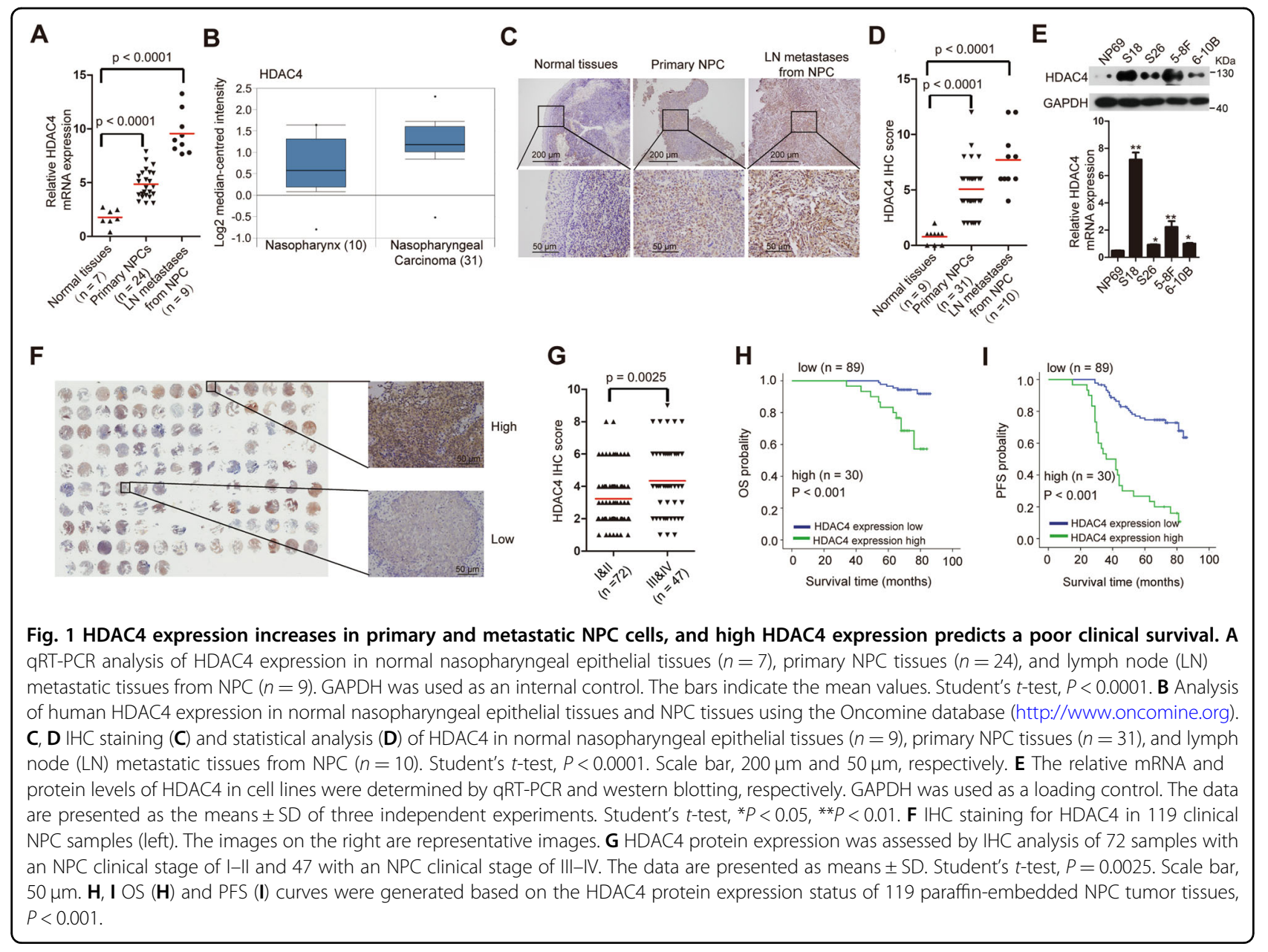

Written informed consent was obtained from all the patients. In total, 74 tumor specimens were collected from patients undergoing NPC resection [tumor percentage $>80 \%$; median age, 45 years; age range, $25-82$ years; male: female ratio, 20:17; clinical staging: I $(n=7)$, II $(n=15)$, III $(n=24)$, and IV $(n=28)]$ between January 2014 and December 2018. Sixteen normal nasopharyngeal epithelium specimens were collected from healthy people (median age, 43 years; age range, $35-73$ years; male:female ratio, 5:3) by resection between January 2013 and December 2017. A human NPC tissue microarray was purchased from Outdo Biotech (Shanghai, China). One hundred nineteen NPC samples were obtained without adjacent normal nasopharynx tissues, and written informed consent was obtained from the patients (median age, 47 years; age range, 20-82 years; male:female ratio, 89:30) with resection between January 2010 and December 2011.

\section{Statistical analysis}

All the in vitro experiments were performed at least three times independently, and the data from each experiment were presented as means $\pm \mathrm{SD}$ or means \pm SEM. All the data followed a normal distribution. Statistical analysis between two groups was performed by Student's $t$-test (two-tailed) with SPSS version 18.0 (IBM Analytics, USA). Spearman's correlation analysis was used to assess the correlation between HDAC4 expression and the clinical stage/grade of the tumor. A $P$ value $<0.05$ was considered to indicate statistical significance in all the cases $\left(^{*}\right)$, and a $P$ value $<0.01$ was considered to indicate strong statistical significance in all the cases $\left({ }^{* *}\right)$.

\section{Results}

HDAC4 expression increases in the primary tissues and LN metastases of NPC, and high HDAC4 levels predict a poor patient prognosis

To further validate the expression level of HDAC4 in NPC according to our microarray data ${ }^{5}$, we first detected its expression in normal nasopharyngeal epithelial tissues, primary NPC tissues, and LN metastases by qRT-PCR. HDAC4 expression was significantly increased in NPC primary tissues and LN metastases compared with that in normal nasopharyngeal epithelial tissues (Fig. 1A). More 


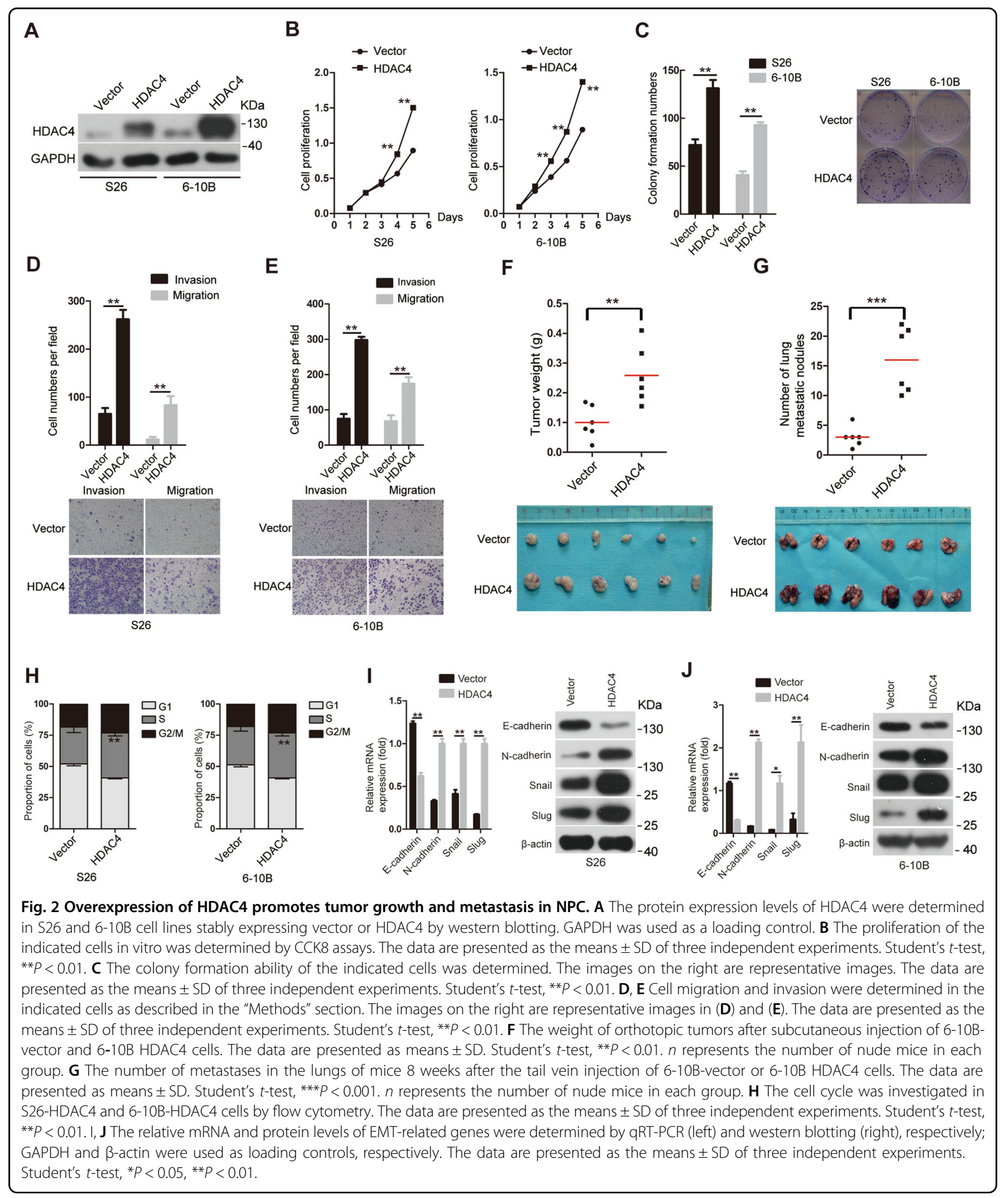

importantly, HDAC4 expression was higher in LN metastases than in primary tumor tissues. Using the Oncomine database, we found that HDAC4 was also higher in NPC tissues than in normal nasopharyngeal epithelial tissues (Fig. 1B). Notably, immunohistochemistry (IHC) demonstrated that the HDAC4 protein levels were substantially higher in primary tumor tissues and LN metastases than in normal nasopharyngeal epithelial 
tissues (Fig. 1C, D). Furthermore, we examined the mRNA and protein levels of HDAC4 by qRT-PCR and western blotting in NP69 cells and four other cell lines (S18 and S26; 5-8F and 6-10B, derived from the NPC cell lines CNE2 and Sune1 and exhibiting high and low metastatic ability, respectively) ${ }^{4}$ (Fig. 1E). Both the mRNA and protein levels of HDAC4 were higher in 5-8F and S18 cells than in 6-10B, S26, and NP69 cells. These results suggested that the expression of HDAC4 was upregulated in primary tissues but increased in metastatic tissues.

To evaluate the prognostic value of HDAC4 expression, we performed immunohistochemical (IHC) staining for HDAC4 using a set of tissue microarrays containing 119 NPC samples (Fig. 1F; Supplementary Fig. 1; Supplementary Table 2). IHC analysis revealed that HDAC4 expression was strongly related to the clinical stage of NPC (Fig. 1G). Correlation analysis also demonstrated that higher expression of HDAC4 was positively associated with a more advanced clinical stage of NPC $(r=$ $0.244, P=0.008$ ). A similar analysis between HDAC4 and histological classification (World Health Organization, WHO) revealed no such association $(r=0.104, P=$ $0.261)$. Via receiver operating characteristic (ROC) analysis, patients were ranked according to the cutoff values of HDAC4 expression and were divided into two groups (low HDAC4 expression group and high HDAC4 expression group). Kaplan-Meier analysis showed that higher HDAC4 expression was significantly correlated with shorter overall survival (OS) and progression-free survival (PFS) times in patients with NPC (Fig. 1H, I). Moreover, to validate the results of our survival analysis, we applied multivariate Cox regression models, which suggested that HDAC4 was a unique indicator of a poor survival prognosis (Supplementary Table 3). These results confirm that the upregulation of HDAC4 is clinically relevant and may be used as an independent prognostic predictor for NPC patients.

\section{Overexpression of HDAC4 promotes tumor growth and metastasis in NPC}

The above results led us to investigate the function of HDAC4 in NPC. Based on the endogenous expression of HDAC4 in NPC cell lines (Fig. 1E), we constructed stably overexpressing HDAC4 in S26 and 6-10B cells, which have low basal HDAC4 expression (Fig. 2A). The results of CCK8 and colony formation assays showed that the cell proliferation and colony formation abilities were significantly increased after ectopic HDAC4 expression compared with those of control cells (Fig. 2B, C). In addition, we employed Transwell assays by plating cells on inserts coated with or without Matrigel and found that the invasion and migration abilities of cells overexpressing HDAC4 were markedly enhanced in vitro compared with those of control cells (Fig. 2D, E). The above results indicate that NPC cell proliferation, migration, and invasion are regulated by HDAC4 in a manner dependent on its expression. To assess the effect of HDAC4 on NPC growth and metastasis in vivo, we established an animal subcutaneous xenograft model by injecting the 6-10Bvector (an empty control vector) or 6-10B-HDAC4 stable cells subcutaneously into nude mice. The tumor weight indicated that the HDAC4 overexpression group showed increased subcutaneous tumor growth compared with the 6-10B-vector cell group (Fig. 2F). Next, we generated an experimental metastasis model in which stable 6-10Bvector or 6-10B-HDAC4 cells were injected into the lateral tail vein of nude mice and assessed after 8 weeks. As expected, HDAC4 overexpression resulted in a significant increase in lung metastasis in the 6-10B-HDAC4 cell group compared with that in the 6-10B-vector cell group (Fig. 2G). Overall, these results suggest that ectopic expression of HDAC4 may contribute to NPC growth and metastasis.

Next, we investigated whether HDAC4 regulated the cell cycle and EMT to promote NPC growth and metastasis. Flow cytometry assays demonstrated that the $\mathrm{S}$ phase showed a significant increase in S26-HDAC4 and 610B-HDAC4 cells compared with that in S26-vector and 6-10B-vector cells, respectively (Fig. 2H; Supplementary Fig. 2A, B). To further confirm the results, we analyzed the expression levels of cyclin D1-CDK4/CDK6 complex members, which promote cell progression through the G1/S checkpoint ${ }^{23}$, by western blotting analysis. As expected, the expression of cyclin D1, CDK4 and CDK6 were significantly increased (Supplementary Fig. 2C, D). These results revealed that HDAC4 induced cell cycle progression by promoting the G1/S phase transition. To explore the mechanism underlying HDAC4-mediated promotion of metastasis, we detected markers of EMT using qRT-PCR and western blotting. HDAC4 overexpression upregulated the expression of $\mathrm{N}$-cadherin, Snail and Slug but downregulated the expression of Ecadherin (Fig. 2I, J), suggesting that ectopic expression of HDAC4 is sufficient to induce EMT.

\section{Knockdown of HDAC4 suppresses cell proliferation, migration, and invasion in vitro and tumor growth in vivo}

To investigate the impact of HDAC4 downregulation on the proliferation and the invasive and migratory abilities of NPC cells, we stably expressed two shRNAs targeting different HDAC4 coding regions (\#1 and \#2) or a scrambled nontargeting shRNA (Scr) in the 5-8F and S18 cell lines, which have a high basal expression of HDAC4, and examined the protein levels of HDAC4 by western blotting (Fig. 3A). The CCK8, colony formation and Transwell assays were performed and demonstrated that S18 and 5-8F cells with HDAC4 knockdown exhibited reduced proliferation, colony formation, invasion and 


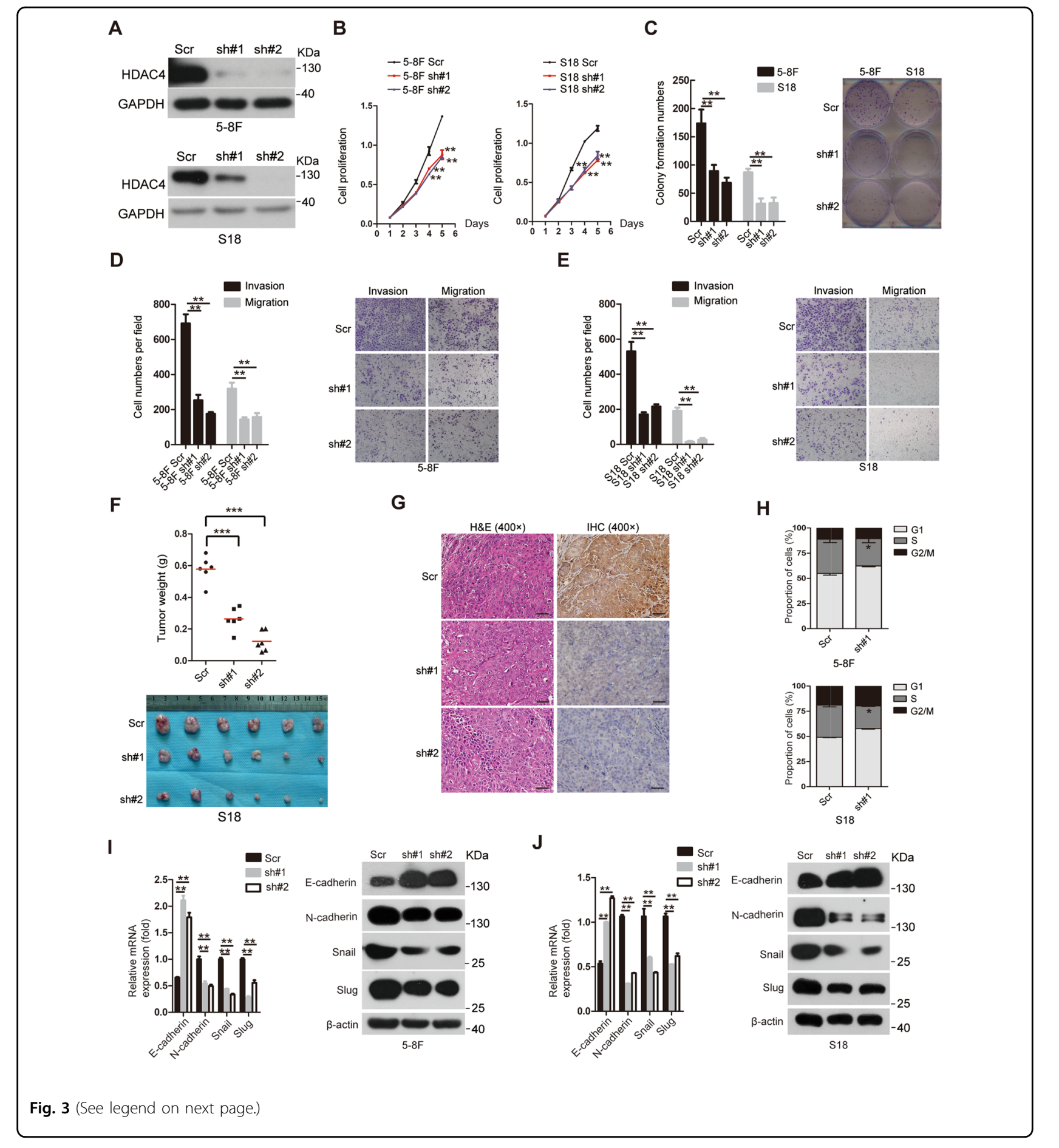

migration abilities compared with S18-Scr and 5-8F-Scr cells, respectively (Fig. 3B-E). In addition, HDAC4 knockdown in $\mathrm{S} 18$ and 5-8F cells resulted in a significant decrease in subcutaneous tumor growth, as shown by measuring the tumor weights (Fig. 3F, G; Supplementary Fig. 3A, B). Taken together, these data indicated that HDAC4 knockdown suppressed the proliferation, invasion and migration abilities of NPC cells in vitro as well as NPC tumor growth in vivo.

We also investigated the cell cycle in stable 5-8FshHDAC4 and S18-shHDAC4 cell lines; 5-8F-Scr and S18-Scr cells were used as the respective control cells. In contrast to the effects of HDAC4 overexpression, G1 arrest was observed in stable 5-8F-shHDAC4 and S18- 
(see figure on previous page)

Fig. 3 Knockdown of HDAC4 suppresses cell proliferation, migration, and invasion in vitro and tumor growth in vivo. A The protein levels of HDAC4 were determined by western blotting in 5-8F and S18 cell lines with or without knockdown. GAPDH was used as a loading control. B The proliferation of the indicated cell lines in vitro was determined by CCK8 assays. The data are presented as the means \pm SD of three independent experiments. Student's $t$-test, ${ }^{* *} P<0.01$. C The colony formation ability of the indicated cells was determined. The images on the right are representative images. The data are presented as the means \pm SD of three independent experiments. Student's $t$-test, ${ }^{* *} P<0.01$. D Cell invasion and migration were determined in the indicated stable cell lines as described in the "Methods" section. The images on the right are representative images. The data are presented as the means \pm SD of three independent experiments. Student's $t$-test, ${ }^{* *} P<0.01$. E Cell invasion and migration were determined in the indicated stable cell lines as described in the "Methods" section. The images on the right are representative images. The data are presented as the means \pm SD of three independent experiments. Student's $t$-test, ${ }^{* *} P<0.01$. F Weight of orthotopic tumors after the subcutaneous injection of S18-Scr cells and S18-shHDAC4\#1\&2 cells. The bottom panel shows an image of the tumors. The data are presented as means \pm SD. Student's $t$-test, ${ }^{* * *} P<0.001$. $n$ represents the number of nude mice in each group. G Representative images of tumors via hematoxylin and eosin (H\&E) and immunohistochemical (IHC) staining for HDAC4. Scale bar, $50 \mu \mathrm{m}$. $\mathbf{H}$ The cell cycle was investigated in 5-8F and S18 cells with or without HDAC4 knockdown by flow cytometry. The data are presented as the means \pm SD of three independent experiments. Student's $t$-test, ${ }^{*} P<0.05$. I, J The relative mRNA and protein levels of EMT-related genes were determined by qRT-PCR (left) and western blotting (right), respectively, in the indicated stable lines. GAPDH and $\beta$-actin were used as loading controls. The data are presented as the means \pm SD of three independent experiments. Student's $t$-test, ${ }^{* *} P<0.01$.

shHDAC4 cell lines compared with that in the corresponding control cells (Fig. 3H; Supplementary Fig. 3C, $\mathrm{D})$, and western blotting demonstrated that the protein levels of cyclin D1, CDK4, and CDK6 were decreased after the knockdown of HDAC4 (Supplementary Fig. 3E, F). These results suggested that HDAC4 knockdown induced cell cycle arrest at the G1 phase partly by inhibiting cyclin D1, CDK4, and CDK6 expression. Furthermore, although HDAC4 knockdown abrogated E-cadherin expression, the expression levels of $\mathrm{N}$-cadherin, Snail, and Slug were decreased at the mRNA and protein levels (Fig. 3I, J). Collectively, our results indicate that HDAC4 facilitates tumor growth and metastasis via promoting G1/S transition and EMT in NPC.

\section{Knockdown of N-CoR abolishes the effects of HDAC4 on the invasion and migration abilities of NPC cells}

Considering that HDAC4 promotes NPC metastasis, we investigated the molecular partners of HDAC4 to elucidate the mechanism by which HDAC4 regulates NPC metastasis. Because the transcriptional corepressor NCoR mediates the functional interaction of HDAC4 and HDAC3 $\left(\right.$ ref. ${ }^{24}$ ), we first examined the interaction among HDAC4, N-CoR, and HDAC3 in 6-10B cells. Endogenous coimmunoprecipitation analysis using anti-HDAC3, antiHDAC4, and anti-N-CoR antibodies showed that HDAC4, N-CoR, and HDAC3 existed in a complex in 610B cells (Fig. 4A). As reported previously, HDAC3 binds to the E-cadherin promoter ${ }^{25,26}$. Luciferase assays were then performed to determine the effect of HDAC3/4 on E-cadherin transcription in NPC cells. Ectopic HDAC3 or HDAC4 expression reduced E-cadherin promoter activity in 6-10B cells (Fig. 4B). Furthermore, ChIP confirmed that HDAC3/4 bound to the E-cadherin promoter in $6-10 \mathrm{~B}$ cells (Fig. 4C). These results suggested that HDAC3/4 was directly bound to the promoter of E-cadherin to repress E-cadherin transcription. Subsequently, we determined whether N-CoR knockdown abolished the effects of HDAC4 on the invasion and migration abilities of NPC cells. S26-HDAC4 and 6-10B-HDAC4 stable cell lines were transfected with or without N-CoR siRNAs (Fig. 4D). The Transwell assays indicated that cotransfection with N-CoR siRNA reversed the increases in the invasion and migration abilities of the S26-HDAC4 and 6-10BHDAC4 cell lines compared with the transfection of S26HDAC4 and 6-10B-HDAC4 cells with siRNA-NC (Fig. 4E, F). Collectively, these results show that the HDAC4/ $\mathrm{N}-\mathrm{CoR} / \mathrm{HDAC} 3$ complex plays a crucial role in the HDAC4-mediated promotion of invasiveness and migration in NPC cells.

\section{Tasquinimod suppresses the HDAC4-induced promotion of cell proliferation in vitro}

Tasquinimod is a high-affinity HDAC4-selective negative allosteric modulator for HDAC4 that can suppress tumor angiogenesis ${ }^{27}$. Considering the above results, we performed CCK8 assays and colony formation to determine whether tasquinimod also inhibits the functional response of NPC cells. Vector cells (S26-vector and 610B-vector), HDAC4-overexpressing cells (S26-HDAC4 and 6-10B-HDAC4), and HDAC4-knockdown cells or control cells were treated with $5 \mu \mathrm{M}$ tasquinimod or DMSO, respectively. Similar to the above results, tasquinimod significantly impaired cell proliferation and colony formation in vector or HDAC4-overexpressing cells compared with those in cells treated with DMSO, as determined by CCK 8 and colony assays. In addition, these phenotypes did not significantly change in HDAC4knockdown cells treated with tasquinimod compared with that in the controls (Fig. 5A-D). Furthermore, the cell cycle in the above cells was analyzed via flow cytometry after treatment with $5 \mu \mathrm{M}$ tasquinimod for $48 \mathrm{~h}$. As expected, the percentage of cells in $\mathrm{S}$ phase was decreased in tasquinimod-treated 6-10B-Vector or 6-10B-HDAC4 


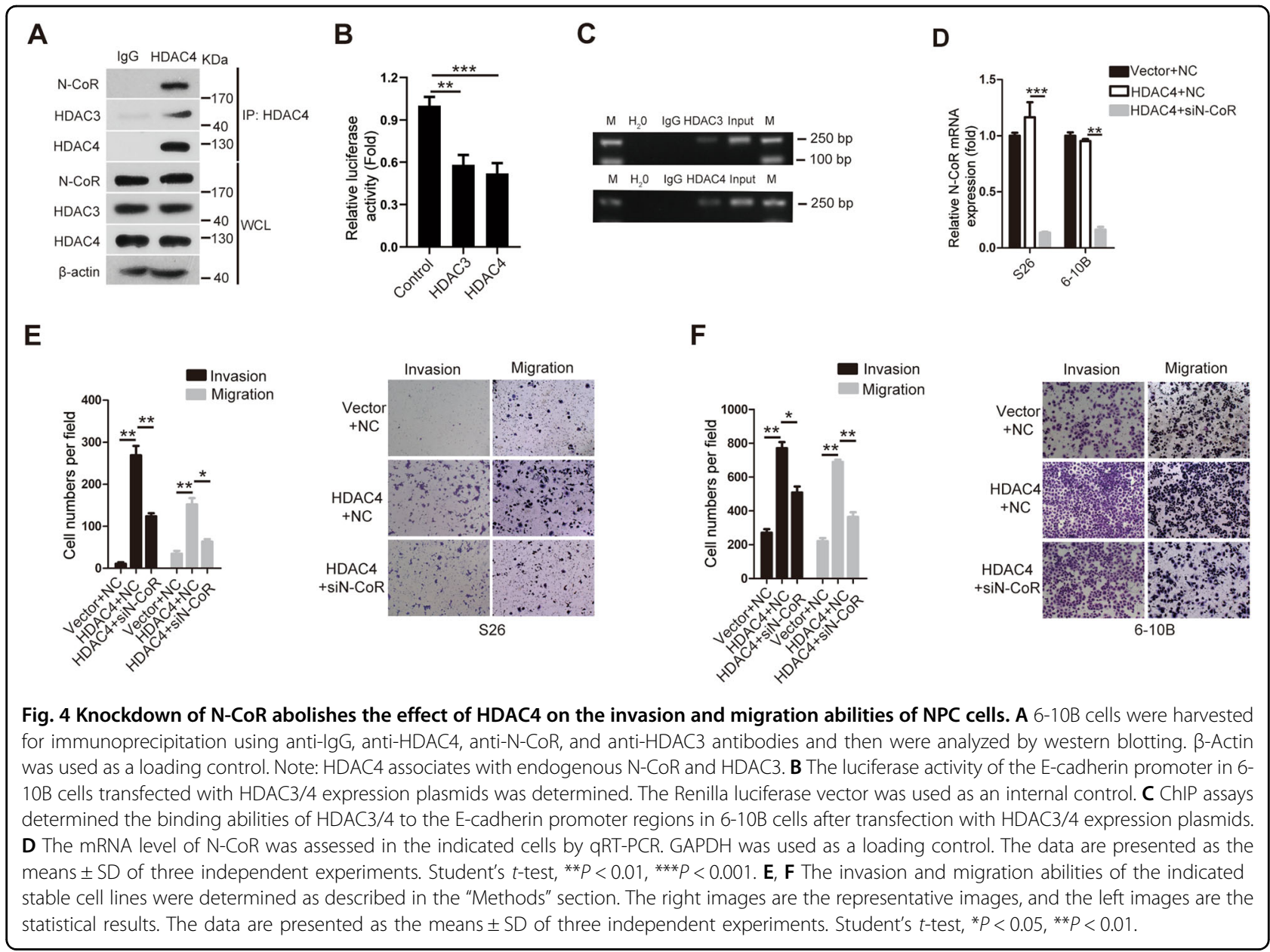

cells compared with that in vehicle-treated cells, respectively. However, the percentage of cells in $\mathrm{S}$ phase was not significantly decreased in tasquinimod-treated $5-8 \mathrm{~F}$ shHDAC4 cells compared with that in vehicle-treated cells (Fig. 5E, F). Tasquinimod dramatically decreased cyclin D1 expression, as demonstrated by western blotting (Fig. 5G). Collectively, our results indicated that tasquinimod suppresses HDAC4-induced cell proliferation via G1/S transition in NPC.

Based on the above observations, we speculated whether tasquinimod could also inhibit other HDACs. To test this hypothesis, we characterized the specificity of tasquinimod based on molecular docking studies using the crystal structures of $\mathrm{Zn}^{2+}$-dependent HDACs that are accessible online [PDB: 6Z2]; 5IX0; 4A69; 4CBT; 6CED; 3ZNR and $5 \mathrm{FCW}]$. According to the docking results, tasquinimod exclusively bound to $\mathrm{Zn}^{2+}$ in the active sites of HDAC4 and HDAC7 among the seven HDACs. Considering the other interactions, such as hydrogen bonds, tasquinimod is predicted to have more affinity with HDAC4, suggesting that tasquinimod mainly affects HDAC4 activity (Supplementary Figs. 4 and 5). However, the effects of tasquinimod on HDAC7 should be further investigated in NPC. In addition, we found no interaction between tasquinimod and HDAC6, and no docking result was obtained using the Ligand Docking module of Schrödinger.

\section{Tasquinimod suppresses the HDAC4-induced promotion of cell migration and invasion in vitro}

To investigate the impact of tasquinimod on the invasive and migratory abilities of NPC cells, Transwell assays were performed. The Transwell assays demonstrated that the invasion and migration abilities were lower in vector or HDAC4-overexpressing cells treated with tasquinimod than in cells treated with DMSO, respectively. However, these phenotypes did not significantly change in HDAC4knockdown cells treated with tasquinimod compared with those in the controls (Fig. 6A-C). Furthermore, tasquinimod abolished HDAC4-mediated EMT, as shown by the detection of EMT markers and observation of phenotypes (Fig. 6D-F), indicating that tasquinimod inhibits HDAC4-induced cell migration and invasion abilities via suppressing the EMT process in NPC. 


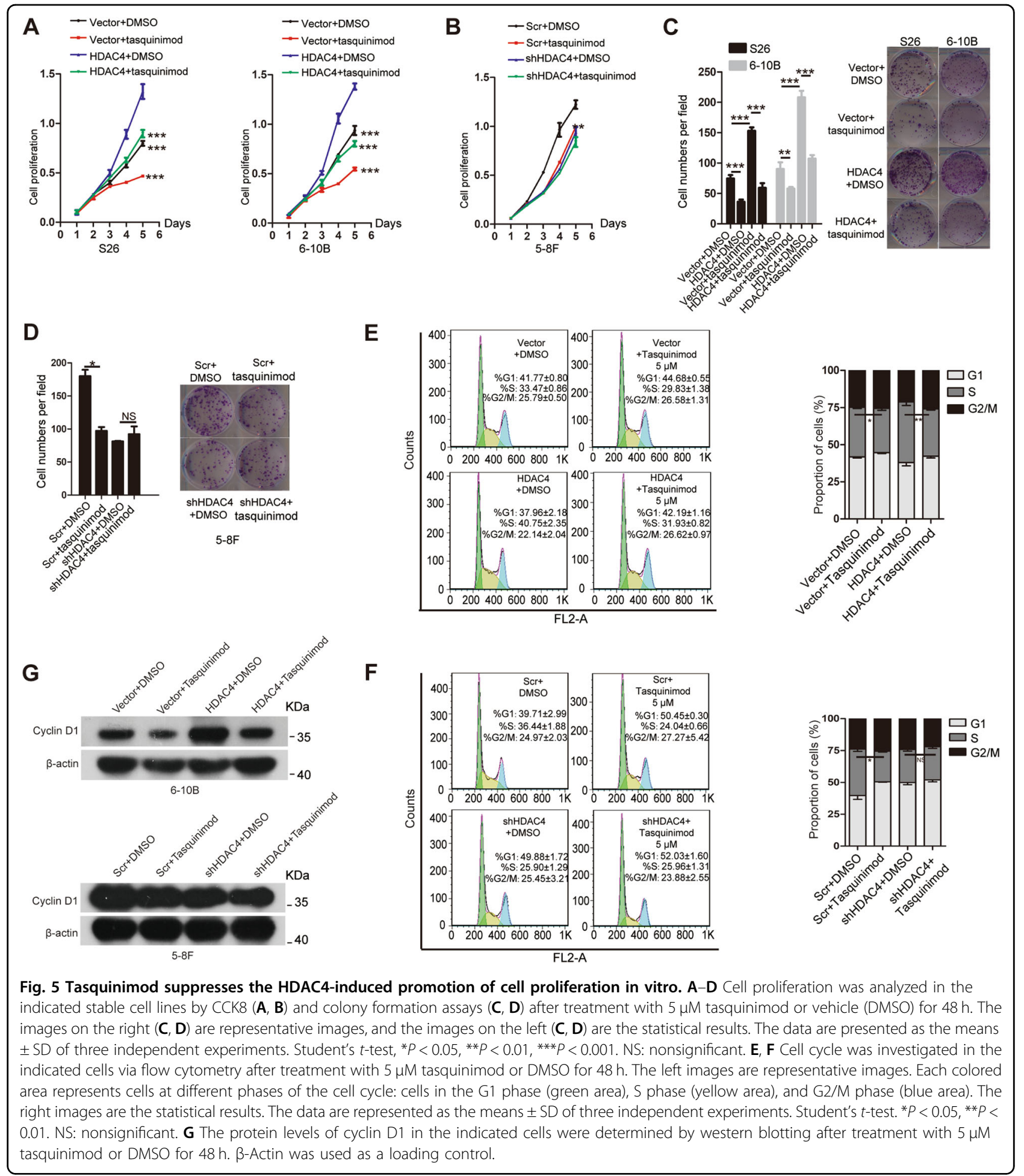

Tasquinimod suppresses the HDAC4-induced promotion of tumor growth in vivo

Next, we sought to assess the efficiency of tasquinimod therapy in vivo. The weights of orthotopic tumors from 6-10B-Vector, 6-10B-HDAC4, 5-8F-Scr, and 5-8FshHDAC4 cells were measured after treatment with tasquinimod or vehicle. Compared with the vehicle, tasquinimod inhibited the weight of subcutaneous xenografts established by injecting vector or HDAC4overexpressing cells (Fig. 7A, B). Furthermore, the reduction in the $5-8 \mathrm{~F}-\mathrm{Scr}$ tumor weight showed a significant difference after tasquinimod treatment compared 


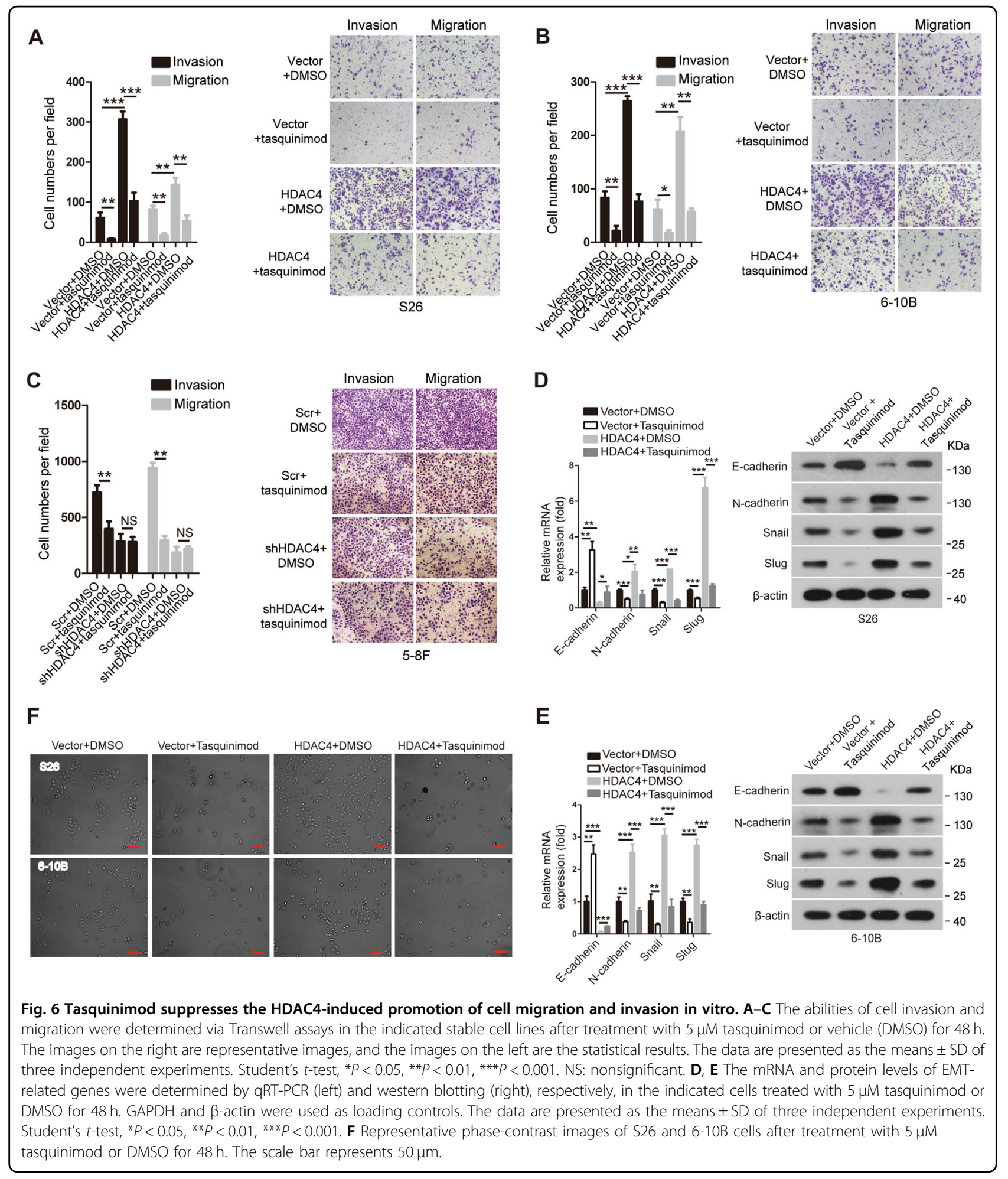

with that after vehicle treatment. However, the 5-8FshHDAC4 tumor weights were barely changed after tasquinimod or vehicle treatment (Fig. 7C, D). Given these results, we concluded that tasquinimod significantly reduced tumor growth in NPC.

\section{Discussion}

NPC has the highest metastasis rate among head and neck cancers, and metastasis is the major cause of treatment failure for NPC patients. While previous work has elegantly demonstrated that HDAC4 plays a vital role in 


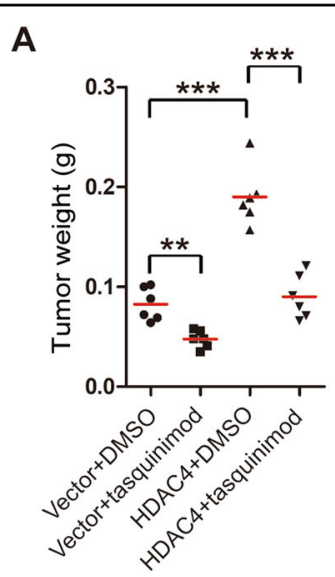

C

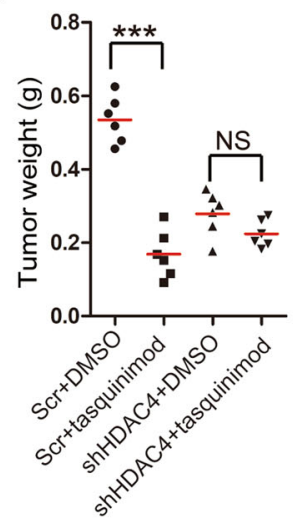

B

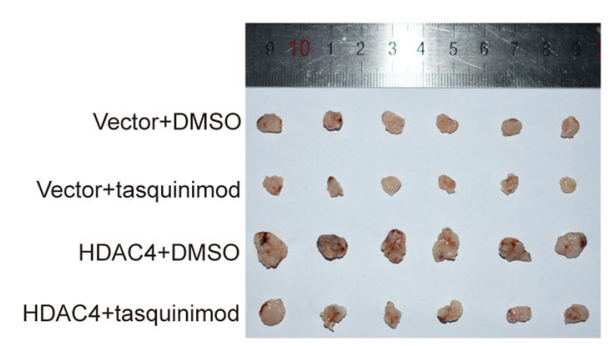

D

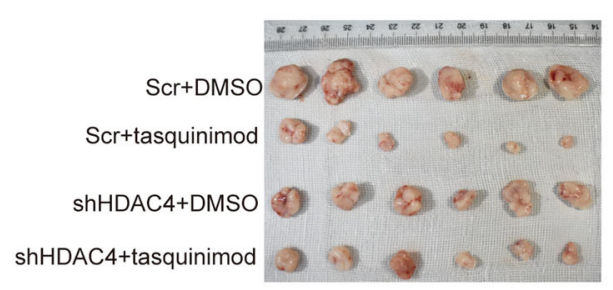

E

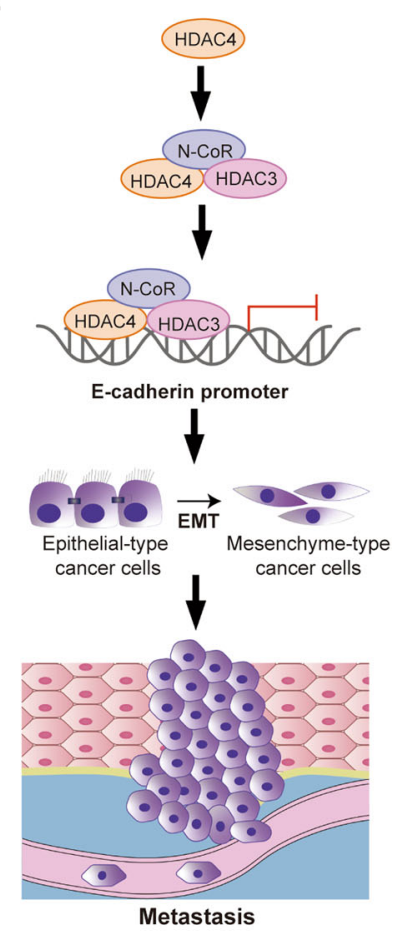

Fig. 7 Tasquinimod suppresses the HDAC4-induced promotion of tumor growth in vivo. A-D The tumor weights (left) in nude mice after the subcutaneous injection of 6-10B-Vector, 6-10B-HDAC4, 5-8F-Scr, or 5-8F-shHDAC4 cells treated with tasquinimod or vehicle (DMSO) (A, C). The image on the right is the tumor image $(\mathbf{B}, \mathbf{D})$. The data are presented as means \pm SD. Student's $t$-test, ${ }^{* *} P<0.01,{ }^{* * *} P<0.001$. NS: nonsignificant. E Proposed model of the role of HDAC4 in promoting tumor metastasis in NPC by binding the promoter of E-cadherin to induce EMT.

cancer development and progression ${ }^{8}$, scant information is available in the literature regarding how HDAC4 is correlated with tumor metastasis. In this study, we demonstrated for the first time that HDAC4 is upregulated in primary and metastatic tissues of NPC. HDAC4 overexpression promoted NPC proliferation, invasion and migration in vitro and enhanced NPC growth and metastasis in vivo. We also showed that HDAC4 overexpression promoted cell cycle G1/S phase transition and EMT. HDAC4 regulates the expression of E-cadherin, Ncadherin, Snail and Slug, and cyclin D1 to promote tumor growth and metastasis in NPC (Fig. 7E).

HDAC4 expression is increased in cancer tissues in various cancers $^{8}$, and our results showed that HDAC4 expression is upregulated not only in primary but also in metastatic tissues of NPC. Therefore, the abnormal expression of HDAC4 demonstrated that it might be closely related to NPC progression. HDAC4 is associated with a poor prognosis and is an independent prognostic factor in esophageal squamous cell carcinoma ${ }^{14}$, and our results showed that a high level of HDAC4 was significantly correlated with a poor prognosis in NPC patients. HDAC4 is an independent prognostic factor in NPC; thus, analyzing the expression of HDAC4 has prognostic value in NPC.

Distant metastasis is the major cause of treatment failure for NPC patients; therefore, understanding the molecular mechanism of NPC is beneficial to the treatment of patients. Previous studies have demonstrated that HDAC4 regulates tumor growth and apoptosis, drug resistance, and cell migration and invasion in colon and hepatocellular carcinoma $^{8}$, and the literature on HDAC4 regulation of cancer metastasis is limited. Moreover, in this study, we provided direct evidence that HDAC4 promotes tumor growth and metastasis in NPC. EMT has been tightly linked with metastasis in diverse types of tumors ${ }^{28-30}$. Our results showed that overexpression or knockdown of HDAC4 increased or decreased the expression of E-cadherin, $\mathrm{N}$ cadherin, Snail and Slug, and may contribute to NPC metastasis. Although HDAC4 promotes the growth of colon cancer cells by suppressing p21 (ref. ${ }^{12}$ ), p21 was not significantly altered in our study (data not shown). 
The development of specific HDAC isoform inhibitors that suppress HDAC activity to achieve clinical results in cancer therapy is one of the most promising approaches. Moreover, understanding the underlying mechanisms by which these inhibitors kill cancer cells is important to effectively treat cancer patients. Tasquinimod, a small molecule that allosterically binds to the regulatory $\mathrm{Zn}^{2+}$ binding domain of HDAC4 (ref. ${ }^{27}$ ), prevented the formation of the HDAC4/N-CoR/HDAC3 repression complex by inhibiting the co-localization of $\mathrm{N}-\mathrm{CoR}$ and HDAC3 (ref. ${ }^{27}$ ). Tasquinimod has demonstrated a good safety profile to treat prostate cancer in phase II and III clinical trials ${ }^{31}$ but has yet to be explored therapeutically in NPC. This study found that tasquinimod exhibited potent inhibitory effects on cell proliferation, colony formation, invasion, and migration in vitro and tumor growth in vivo in NPC, suggesting that tasquinimod is a potential candidate for NPC treatment. In addition, molecular docking analysis showed inhibitory activity of tasquinimod against $\mathrm{HDAC7}$, but further studies are necessary to verify this observation. A study indicated that the use of tasquinimod had several adverse effects for the treatment of metastatic castration-resistant prostate cancer (mCRPC), such as anemia, back pain, and insomnia ${ }^{32}$. However, tasquinimod has a well-tolerated safety profile suitable for chronic use, which is an option for men before taxane chemotherapy to delay progression and symptomatic disease ${ }^{33}$. Therefore, toxicity and dosage studies must be performed before treatment in patients with NPC. Research has shown that HDAC inhibitors might act against specific HDACs (isoform-selective inhibitors) and all HDAC types (pan-inhibitors) ${ }^{34}$. Considering the potency and selectivity of HDAC inhibitors, effective inhibitors of HDAC4 warrant further study and development. In addition, the combination with other drugs may be more effective in treating NPC patients. However, further experiments are required to verify this hypothesis.

In conclusion, our present study highlighted an important role of HDAC4 in promoting tumor growth and metastasis in NPC. HDAC4 may serve as a valuable target for treating patients with NPC.

\section{Acknowledgements}

We thank the members of the laboratory for their helpful comments on the manuscript. We thank American Journal Experts (www.aje.com) for English language editing.

\section{Funding}

This study was supported by the National Science Foundation of China (grant no. 81660449 to Y.S.).

\footnotetext{
Author details

${ }^{1}$ Jiangxi Key Laboratory of Cancer Metastasis and Precision Treatment, Department of Center Laboratory, The Third Affiliated Hospital of Nanchang University, Nanchang 330008, China. ${ }^{2}$ Department of Oncology, Tongji
}

Huangzhou Hospital of Huazhong University of Science and Technology, Hubei, People's Republic of China. ${ }^{3}$ Department of Surgery, Pathology and Oncology, University of Western Ontario, London N6G5H5, Canada

\section{Author contributions}

Y.S. and C.C. made substantial contributions to the conception and design of the present study. C.C., J.Y. and S.W.L. developed the methodology and acquired, analyzed, and interpreted the data. G.F.H., C.X.L., W.P.M. and Y.S. wrote and reviewed the manuscript. Y.S. supervised the study. All the authors read and approved the final version of the manuscript.

\section{Conflict of interest}

The authors declare that they have no conflict of interest.

\section{Ethics approval and consent to participate}

The research was approved by the Ethics Committee of the Third Affiliated Hospital of Nanchang University.

\section{Publisher's note}

Springer Nature remains neutral with regard to jurisdictional claims in published maps and institutional affiliations.

Supplementary information The online version contains supplementary material available at https://doi.org/10.1038/s41419-021-03417-0.

Received: 22 July 2020 Revised: 6 January 2021 Accepted: 8 January 2021 Published online: 01 February 2021

\section{References}

1. Chua, M. L. K. Wee, J. T. S., Hui, E. P. \& Chan, A. T. C. Nasopharyngeal carcinoma. Lancet 387, 1012-1024 (2016).

2. Sang, Y., Cheng, C., Zeng, Y. X. \& Kang, T. Snail promotes metastasis of nasopharyngeal carcinoma partly by down-regulating TEL2. Cancer Commun. 38, 58 (2018).

3. You, R. et al. The changing therapeutic role of chemo-radiotherapy for locoregionally advanced nasopharyngeal carcinoma from two/three-dimensional radiotherapy to intensity-modulated radiotherapy: a network meta-analysis. Theranostics 7, 4825-4835 (2017).

4. Wang, L. et al. Down-regulation of prostate stem cell antigen (PSCA) by Slug promotes metastasis in nasopharyngeal carcinoma. J. Pathol. 237, 411-422 (2015).

5. Sang, Y. et al. TEL2 suppresses metastasis by down-regulating SERPINE1 in nasophanyngeal carcinoma. Oncotarget 6, 29240-29253 (2015).

6. Luan, Y., Li, J., Bernatchez, J. A. \& Li, R. Kinase and histone deacetylase hybrid inhibitors for cancer therapy. J. Med. Chem. 62, 3171-3183 (2019).

7. Parra, M. Class lla HDACs - new insights into their functions in physiology and pathology. FEBS J. 282, 1736-1744 (2015).

8. Wang, Z., Qin, G. \& Zhao, T. C. HDAC4: mechanism of regulation and biological functions. Epigenomics 6, 139-150 (2014).

9. Amodio, N. et al. Therapeutic targeting of miR-29b/HDAC4 epigenetic loop in multiple myeloma. Mol. Cancer Ther. 15, 1364-1375 (2016).

10. Halkidou, K., Cook, S., Leung, H. Y., Neal, D. E. \& Robson, C. N. Nuclear accumulation of histone deacetylase 4 (HDAC4) coincides with the loss of androgen sensitivity in hormone refractory cancer of the prostate. Eur. Urol. 45, 382-389 (2004)

11. Ahmad, A. et al. Functional role of miR-10b in tamoxifen resistance of ERpositive breast cancer cells through down-regulation of HDAC4. BMC Cancer 15, 540 (2015).

12. Wilson, A. J. et al. HDAC4 promotes growth of colon cancer cells via repression of p21. Mol. Biol. Cell 19, 4062-4075 (2008).

13. Marampon, F. et al. HDAC4 and HDAC6 sustain DNA double strand break repair and stem-like phenotype by promoting radioresistance in glioblastoma cells. Cancer Lett. 397, 1-11 (2017).

14. Zeng, L. S. et al. Overexpressed HDAC4 is associated with poor survival and promotes tumor progression in esophageal carcinoma. Aging 8, 1236-1249 (2016). 
15. Ren, G. et al. Recruitment of HDAC4 by transcription factor $Y Y 1$ represses HOXB13 to affect cell growth in AR-negative prostate cancers. Int. J. Biochem. Cell Biol. 41, 1094-1101 (2009).

16. Vallabhapurapu, S. D. et al. Transcriptional repression by the HDAC4-RelB-p52 complex regulates multiple myeloma survival and growth. Nat. Commun. $\mathbf{6}$, 8428 (2015).

17. Zhang, R. et al. TRIM11 facilitates chemoresistance in nasopharyngeal carcinoma by activating the beta-catenin/ABCC9 axis via p62-selective autophagic degradation of Daple. Oncogenesis 9, 45 (2020).

18. Livak, K. J. \& Schmittgen, T. D. Analysis of relative gene expression data using real-time quantitative PCR and the 2(-Delta Delta C(T)) Method. Methods 25 402-408 (2001).

19. Sang, $Y$. et al. LncRNA PANDAR regulates the G1/S transition of breast cancer cells by suppressing p16(INK4A) expression. Sci. Rep. 6, 22366 (2016).

20. Sang, Y. et al. Histone deacetylase 7 inhibits plakoglobin expression to promote lung cancer cell growth and metastasis. Int. J. Oncol. 54, 1112-1122 (2019).

21. Hong, J. et al. CHK1 targets spleen tyrosine kinase $(L)$ for proteolysis in hepatocellular carcinoma. J. Clin. Invest. 122, 2165-2175 (2012).

22. Remmele, W. \& Stegner, H. E. Recommendation for uniform definition of an immunoreactive score (IRS) for immunohistochemical estrogen receptor detection (ER-ICA) in breast cancer tissue. Pathologe 8, 138-140 (1987).

23. Semczuk, A. \& Jakowicki, J. A. Alterations of pRb1-cyclin D1-cdk4/6-p16(INK4A) pathway in endometrial carcinogenesis. Cancer Lett. 203, 1-12 (2004).

24. Fischle, W. et al. Enzymatic activity associated with class II HDACs is dependent on a multiprotein complex containing HDAC3 and SMRT/N-CoR. Mol. Cell 9 45-57 (2002).
25. Yao, R. et al. PRMT7 induces epithelial-to-mesenchymal transition and promotes metastasis in breast cancer. Cancer Res 74, 5656-5667 (2014).

26. Wu, M. Z. et al. Interplay between HDAC3 and WDR5 is essential for hypoxiainduced epithelial-mesenchymal transition. Mol. Cell 43, 811-822 (2011).

27. Isaacs, J. T. et al. Tasquinimod Is an Allosteric Modulator of HDAC4 survival signaling within the compromised cancer microenvironment. Cancer Res. 73, 1386-1399 (2013).

28. Mao, Z. et al. CXCL5 promotes gastric cancer metastasis by inducing epithelial-mesenchymal transition and activating neutrophils. Oncogenesis 9,63 (2020)

29. $\mathrm{Yi}, \mathrm{H}$. et al. Integrative multi-omics analysis of a colon cancer cell line with heterogeneous Wnt activity revealed RUNX2 as an epigenetic regulator of EMT. Oncogene 39, 5152-5164 (2020).

30. Xiong, Y. et al. WFDC2 suppresses prostate cancer metastasis by modulating EGFR signaling inactivation. Cell Death Dis. 11, 537 (2020).

31. Lang, C. et al. Single-cell sequencing of iPSC-dopamine neurons reconstructs disease progression and identifies HDAC4 as a regulator of parkinson cell phenotypes. Cell Stem Cell 24, 93-106 e106 (2019).

32. Gong, P. et al. Efficacy of tasquinimod in men with metastatic castrationresistant prostate cancer: a meta-analysis of randomized controlled trials. Medicine 97, e13204 (2018).

33. Mehta, A. R. \& Armstrong, A. J. Tasquinimod in the treatment of castrateresistant prostate cancer-current status and future prospects. Ther. Adv. Urol. 8, 9-18 (2016).

34. Eckschlager, T., Plch, J., Stiborova, M. \& Hrabeta, J. Histone deacetylase inhibitors as anticancer drugs. Int. J. Mol. Sci. 18,1414 (2017). 